\title{
Distribución del tamaño de partícula de la tetracarboxifenilporfirina de hierro (III) adsorbida sobre dióxido de silicio por dispersión dinámica de luz
}

\author{
Carlos Enrique Diaz Uribe*, William Andrés Vallejo ${ }^{\star \star}$, Jarol Miranda \\ Grupo de Investigación en Fisicoquímica Aplicada y Estudios Ambientales. Laboratorio \\ de Fotoquímica y Fotobiología. Programa de Química. Facultad de Ciencias Básicas. \\ Universidad del Atlántico. Kilómetro 7 Vía Puerto Colombia. Barranquilla - Colombia.
}

FECHA DE ENTREGA: 14 DE FEBRERo DE 2013

FECHA DE APROBACIÓN: 7 DE MARZO DE 2013

\begin{abstract}
Resumen La distribución del tamaño de partícula es una propiedad fundamental para caracterizar sistemas de partículas en suspensión. Las porfirinas son sensibilizadores esenciales para diversas aplicaciones fotoquímicas, sin embargo estos sensibilizadores tienden a formar agregados en solución acuosa, afectando sus propiedades fotofísicas, lo que hace necesaria su inmovilización. En este trabajo se estudió la distribución del tamaño de partícula de la tetracarboxifenilporfirina de hierro (III) adsorbida sobre dióxido de silicio por la técnica de dispersión dinámica de luz. La estabilidad del proceso de adsorción depende del valor del $\mathrm{pH}$, es por esto, que se estudió a un pH de 3 y 5 . Se encontró un solo tamaño de partícula a $\mathrm{pH}=3$ indicando la estabilidad de la porfirina sobre la silica, mientras que a $\mathrm{pH}=5$, dos tipos de partículas fueron encontradas. Los resultados indican que la técnica de dispersión dinámica de luz es adecuada para determinar la estabilidad de estos sistemas.
\end{abstract}

\begin{abstract}
Particle size distribution is a critical property for characterizing particles in suspension systems. Porphyrins are sensitizers essential for different photo-chemical applications, however these compounds form aggregates in aqueous solution, this phenomen changes its photophysical properties, due to this necessary to inmobilize the porphyrins. In this work, the particle size distribution of tetracarboxyphenyl porphyrin iron (III) adsorbed on silicon dioxide was studied by the technique of dynamic light scattering. Results indicate that the stability of the adsorption process depends on the $\mathrm{pH}$ value. It was observed a single particle size at $\mathrm{pH}=3$ indicating stability of the porphyrin on silica, while at $\mathrm{pH}=5$, two types of particles were found. The results indicate that the technique of dynamic light scattering is suitable for determining the stability of these systems.
\end{abstract}

Palabras Clave: porfirina, silica, DLS, tamaño de partícula.

Keywords: porphyrin, silica, DLS, particle size.

* carlosdiaz@mail.uniatlantico.edu.co

** williamvallejo@mail.uniatlantico.edu.co 


\section{Introducción}

Las porfirinas son una de las unidades químicas vitales esenciales para la vida en la tierra y participan en una serie de procesos biológicos de gran relevancia para los seres vivos, como son la catálisis bioquímica, la transferencia electrónica, el transporte y activación de oxígeno y la fotosíntesis [12]. Las porfirinas son utilizadas como sensibilizadores debido a su alta absorción en la región visible del espectro electromagnético, estos compuestos son eficientes en las reacciones de oxidación catalítica y generan productos menos tóxicos y ambientalmente más amigables [3. Sin embargo, las porfirinas tienden a formar agregados en solución acuosa esto ocasiona que propiedades fotofísicas sean afectadas y puedan llegar a disminuir 44. Su inmovilización bloquea la formación de agregados, facilita la reutilización del sensibilizador, la separación de los productos de la reacción, y la utilización de diferentes solventes [56]. Una de las matrices más utilizadas para inmovilizar este tipo de compuestos es el dióxido de silicio [789. Las porfirinas pueden anclarse o adsorberse en la superficie del $\mathrm{SiO}_{2}$ mediante un enlace covalente [10], enlace coordinado [11, interacción iónica [12], intercalación [13], y adsorción [14]. Cada uno de estos tipos de interacciones entre el sensibilizador y el soporte genera ventajas dependiendo de la fortaleza de la unión al soporte, la preparación, la aplicación general y la estabilidad.

Hay un considerable interés en insertar complejos macrocíclicos dentro de materiales amorfos porosos por simple adsorción para diversas aplicaciones [1516]. La mayor ventaja de encapsular el sensibilizador es el control del medio de reacción y sus condiciones de preparación [17. Además, al no estar enlazados químicamente no se ve afectada la planaridad del sensibilizador y por ende las propiedades fotofísicas. Porfirinas con sustituyentes como $-\mathrm{NH}_{2},-\mathrm{COOH},-\mathrm{OH}$ y $-\mathrm{SO}_{3} \mathrm{H}$, son ideales para la inserción en los poros de la silica [18].

El valor del $\mathrm{pH}$ en solución es un parámetro fundamental que previene la lixiviación de un sensibilizador, es por esto, que en este trabajo se estudió la distribución del tamaño de partícula de la tetracarboxifenilporfirina de hierro (III) adsorbida sobre dióxido de silicio a un pH de 3 y 5 por la técnica de dispersión dinámica de luz. La técnica de dispersión de luz dinámica permite determinar el tamaño de partículas en suspensión hasta rangos del orden de los nanómetros, el uso del movimiento Browniano de las partículas en suspensión permite alcanzar estos límites de sensibilidad, adicionalmente este método no altera de ninguna manera la matriz de estudio debido a que es una técnica no destructiva. Los resultados indican que la técnica de dispersión dinámica de luz es adecuada para determinar la estabilidad de los sensibilizadores soportados a la silica.

\section{Metodología}

\subsection{Síntesis y caracterización}

La tetracarboxifenilporfirina sin metal se sintetizó añadiendo $30 \mathrm{mmol}$ de pirrol previamente destilado a una mezcla de 4-carboxibenzaldehido (30 mmol), ácido propiónico $(105 \mathrm{~mL})$ y nitrobenceno $(45 \mathrm{~mL})$. La mezcla se calentó a $120{ }^{\circ} \mathrm{C}$ 
durante $1 \mathrm{~h}$. El solvente se removió por destilación al vacío y el sólido obtenido se disolvió con una solución de $\mathrm{NaOH}(0,1 \mathrm{M})$. La porfirina se precipitó con una solución de $\mathrm{HCl}$ 0,1 M, se disolvió en etanol y se cristalizó por evaporación del solvente [19].

La metaloporfirina se preparó colocando a reflujo la TCCPP $(0.33 \mathrm{mmol})$ con cloruro de hierro (III) en cantidades correspondientes a 1,82 mmol, en dimetilformamida $(70 \mathrm{~mL})$ durante $2 \mathrm{~h}$. La dimetilformamida se removió por destilación y la TCPPFe se precipitó adicionando agua. El precipitado se disolvió en una solución de $\mathrm{NaOH}(0,1 \mathrm{M})$ y se recristalizó con una solución de $\mathrm{HCl}(0,1$ $\mathrm{M})$. Finalmente, la porfirina se filtró y secó a temperatura ambiente.

La TCPPFe/ $/ \mathrm{SiO}_{2}$ se preparó según el siguiente procedimiento: se agregó $0.08438 \mathrm{~g}$ de TCPPFe a una mezcla de $(20 \mathrm{~mL}$ de etanol absoluto, $3 \mathrm{~mL}$ de tetraetoxisilano (TEOS) y $0.5 \mathrm{~mL}$ de agua destilada) a $\mathrm{pH}=3$ (ajustado con $\mathrm{HNO}_{3}$ ). Se dejó en reflujo por 24 horas, el solvente se rotoevaporó y el sólido se secó a temperatura ambiente [20].

Los espectros UV-vis de la TCPP y TCPPFe en etanol se registraron usando un espectrofotómetro Hewlett-Packard 8453. Los espectros de reflectancia difusa UV-vis de la porfirina adsorbida se registraron utilizando un espectrofotómetro Lambda 4 Perkin-Elmer equipado con una esfera de integración, empleando sulfato de bario como matriz de referencia.

\subsection{Caracterización del tamaño de partícula por dispersión dinámica de luz}

El tamaño de partícula en suspensión fue determinado mediante la técnica de Dispersión Dinámica de Luz (Espectroscopia de Correlación Fotónica). El equipo utilizado fue un Zetasizer versión 6.2 de Malvern Instruments Ltda. Para el análisis se tomó $1 \mathrm{~g} / \mathrm{L}$ de $\mathrm{TCPPFe} / \mathrm{SiO}_{2}$ a $\mathrm{pH}=3$ y $\mathrm{pH}=5$ en agua como dispersante. Todos los experimentos se midieron mediante dispersión dinámica, el cual cuantifica las fluctuaciones de la intensidad de la dispersión en función del tiempo. La distribución de intensidad vista en el programa DLS se obtuvo por el análisis de la función de correlación por el análisis del menor de los cuadrados no negativo.

\section{Resultados y discusión}

\subsection{Espectrofotometría}

El espectro UV-vis de la TCPP sintetizada se muestra en la (figura 1), en él se observa la banda de absorción máxima denominada banda Soret a $419 \mathrm{~nm}$ (transición $\mathrm{a}_{1 \mathrm{u}}(\pi)-\mathrm{e}_{\mathrm{g}}^{*}(\pi)$, y 4 bandas Q $(514,548,588$ y $645 \mathrm{~nm})$ características de la porfirina no metálica correspondientes a transiciones $\mathrm{a}_{2 \mathrm{u}}(\pi)-\mathrm{e}_{\mathrm{g}}^{*}(\pi)$ [21]; estas señales se maximizan dentro de la Figura 1.

El espectro UV-vis de la TCPPFe (Figura 1) exhibe la banda Soret a 413 $\mathrm{nm}$ y 1 banda Q en $534 \mathrm{~nm}$. La disminución del número de bandas Q es típico 


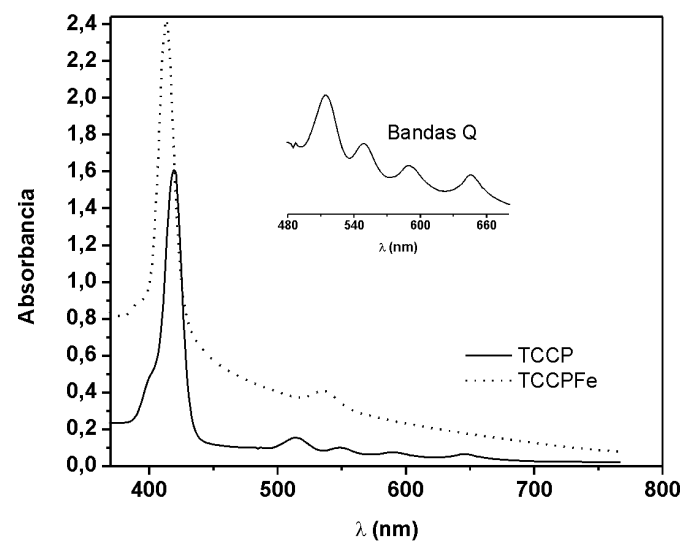

Figura 1. Espectro UV-vis de la TCPP y de la TCPPFe.

de las metaloporfirinas. Cuando el ión metálico se coordina con los átomos de nitrógeno del macrociclo, la simetría de la molécula se incrementa y el número de bandas Q decrece 22. La metalación de las porfirinas produce desplazamiento de la bandas Soret y Q por la interacción del metal con la nube electrónica $\pi$ de los macrociclos. En el espectro de la TCPPFe se observa un desplazamiento de las bandas de absorción hacia el azul, posiblemente debido a la desestabilización de la nube electrónica $\pi$ del ligando inducida por el metal e incremento de la energía de transición $[22$.

\subsection{Reflectancia difusa}

El espectro de reflectancia difusa de la TCPPFe/ $\mathrm{SiO}_{2}$ se muestra en la Figura 2 Las bandas de absorción de TCPPFe adsorbida sobre $\mathrm{SiO}_{2}$ son anchas y de menor intensidad con respecto al espectro de TCPPFe (III), y muestran un corrimiento hacia la región del rojo que puede atribuirse a la distorsión de los anillos de porfirina; el desplazamiento hacia el rojo generalmente se observa cuando las porfirinas son inmovilizadas sobre soportes inorgánicos [2324]. En el caso de la TCPPFe adsorbida al $\mathrm{SiO}_{2}$, la banda Soret es desplazada de 419 a $427 \mathrm{~nm}$ y la banda Q de igual manera es desplazada de 534 a $542 \mathrm{~nm}$, corroborando que el proceso de inmovilización se ha realizado.

\subsection{Caracterización del tamaño de partícula por dispersión dinámica de luz-DLS}

Para analizar el tamaño de partícula en la solución se tomó 1g/L de TCPPFe/ $\mathrm{SiO}_{2}$ a $\mathrm{pH}=3 \mathrm{y} \mathrm{pH}=5$ en agua como dispersante. El análisis se realizó mediante dispersión dinámica, el cual mide las fluctuaciones de la intensidad de la dispersión en función del tiempo. La Figura 3 muestra el coeficiente de correlación de la 


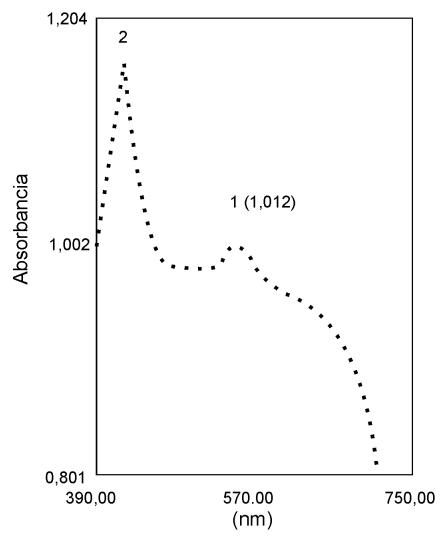

Figura 2. Espectro de reflectancia difusa TCPPFe(III)/ $/ \mathrm{SiO}_{2}$.

intensidad de luz dispersada por las partículas de TCPPFe/ $\mathrm{SiO}_{2}$ a pH 3 y pH 5 . El punto en que la correlación de la señal empieza a caer proporciona información del diámetro promedio y el ángulo de caída permite observar la dispersidad de la distribución de las partículas. En ambos casos se obtiene un ángulo similar y una línea de base corta, regular y con poco ruido, lo que permite inferir la baja polidispersidad en ambos casos.

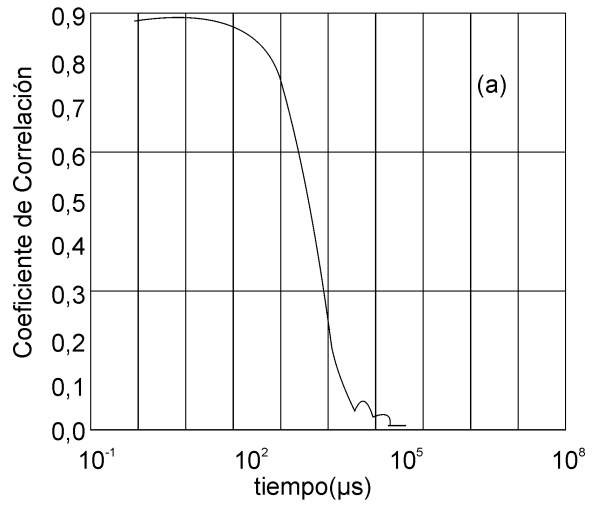

(a)

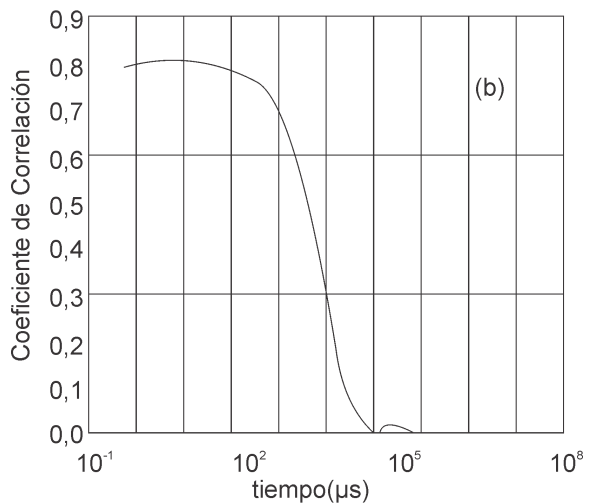

(b)

Figura 3. Coeficiente de correlación de la intensidad de luz dispersada: (a) $\mathrm{pH}=3 \mathrm{y}$ (b) $\mathrm{pH}=5$.

La Figura 4 muestra la calidad del ajuste de cumulantes (autocorrelación) a los datos medidos indicando que el diámetro calculado y la dispersidad obtenida 
para las partículas medidas son fiables. En ellas se puede ver que las funciones de autocorrelación de la intensidad de luz dispersada disminuyen más rápidamente después de los $1000 \mu$ s.

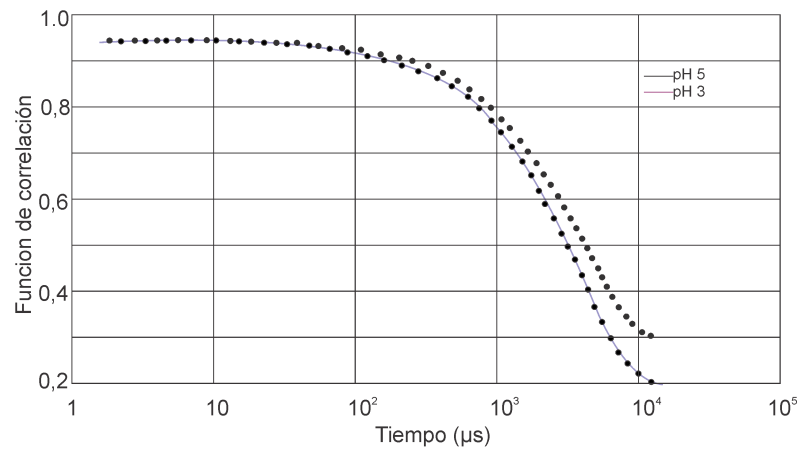

Figura 4. Función de autocorrelación de luz dispersada (a) $\mathrm{pH}=3$ (b) $\mathrm{pH}=5$.

El análisis de DLS correspondiente a la intensidad muestra una banda a $\mathrm{pH}=3$ y dos bandas a $\mathrm{pH}=5$ (Figura 5), este último sugiere la formación de dos grupos de partículas: las primeras corresponden a un grupo de partículas pequeñas y las otras partículas con mayores tamaños posiblemente como resultado de agregación de la porfirina por lixivación del sensibilizador.

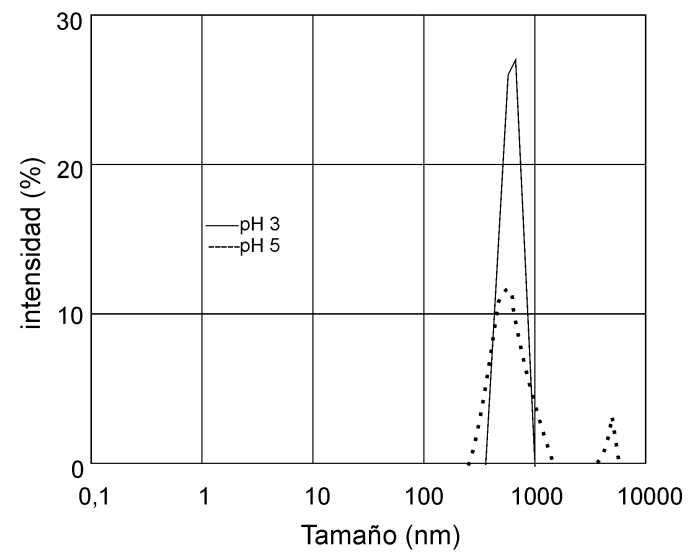

Figura 5. Estudio de DLS en intensidad a $\mathrm{pH}=3$ y $\mathrm{pH}=5$.

Las partículas mayores, dispersan más la trayectoria del láser y consecuentemente pueden generar cierta intensidad, así mismo la cantidad de este tipo de 
partículas presente en el volumen puede ser pequeño. En la Figura 6 se muestran las gráficas del tamaño de partícula en función del número de partículas. Se puede observar, que la cantidad de partículas de mayor tamaño es tan pequeña que no aparecen en el gráfico (solo se observa una señal), es decir, las partículas de menor tamaño $\mathrm{TCPPFe} / \mathrm{SiO}_{2}$ son predominantes, inclusive a $\mathrm{pH}=5$. Sin embargo se observa que la señal a pH 5,0 es más ancha que a pH 3,0 indicando que se presenta mayor dispersidad debido a la presencia de dos tipos de partículas con diámetros diferentes.

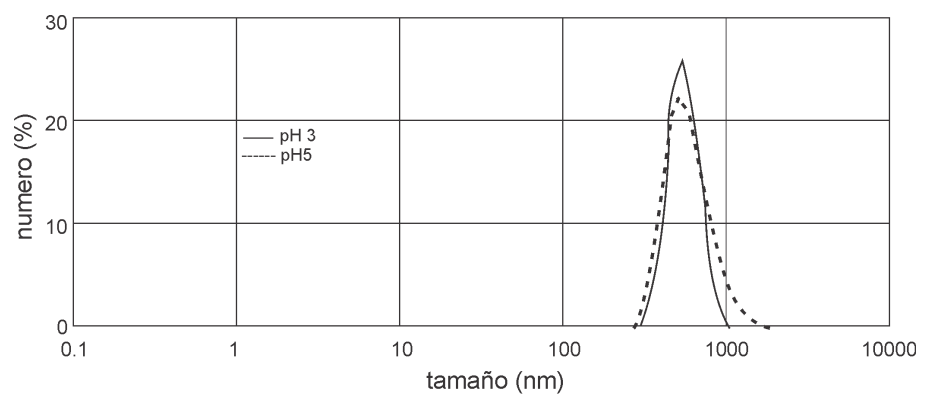

Figura 6. Distribución del número de partículas (a) $\mathrm{pH}=3$ (b) $\mathrm{pH}=5$.

Las gráficas de volumen en función del tamaño de partícula confirman que a $\mathrm{pH}=3$ solo hay un mismo tipo de partícula, mientras que a $\mathrm{pH}$ mayores hay dos tipos de partículas, posiblemente agregados de porfirina lixiviada. La Figura 7 muestra la variación del porcentaje en volumen con respecto al tamaño de las partículas presentes en función del $\mathrm{pH}$. De nuevo se corrobora que a $\mathrm{pH}=3$ solo se presenta un tipo de partícula; por su parte a $\mathrm{pH}=5$ se observan dos picos indicando la presencia de dos tipos de partícula. La altura de la señal para las partículas de mayor tamaño es mayor que para las de menor tamaño debido a su diámetro de partícula a pesar que se encuentren en muy baja proporción.

\section{Conclusiones}

Mediante la técnica de dispersión dinámica de luz, se encontró un solo tipo de partícula a $\mathrm{pH}=3$ del sistema tetracarboxifenilporfirina de hierro (III) adsorbida en dióxido de silicios en dispersión acuosa, esto indica la estabilidad de la porfirina sobre la silica a este valor de $\mathrm{pH}$, mientras que a $\mathrm{pH}=5$, dos tipos de partículas fueron encontradas, posiblemente a la lixiviación de sensibilizador y formación de agregados. Este resultado es relevante, ya que indica que el sistema $\mathrm{TCPPFe} / \mathrm{SiO}_{2}$ puede ser utilizado en diferentes procesos de fotocatálisis para oxidación de colorantes. Finalmente los resultados indican que la técnica de dispersión dinámica de luz es adecuada para determinar la estabilidad de estos sistemas. 


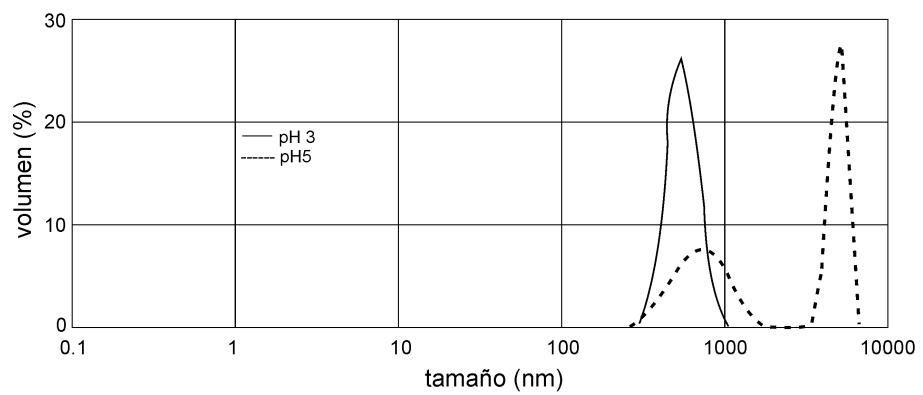

Figura 7. Distribución del volumen en función del tamaño de partícula.

Agradecimientos: Los autores agradecen al profesor Fernando Martínez Ortega de la Universidad Industrial de Santander por el análisis de DLS.

\section{Referencias}

1. Adilina, I., Hara, T., Ichikuni, N., Shimazu, S.: Oxidative cleavage of isoeugenol to vanillin under molecular oxygen catalysed by cobalt porphyrin intercalated into lithium taeniolite clay. Journal of Molecular Catalysis A: Chemical. 361 72-79. (2012)

2. Lahaye, D., Groves, J.: Modeling the haloperoxidases: Reversible oxygen atom transfer between bromide ion and an oxo-Mn(V) porphyrin. Journal of Inorganic Biochemistry. 101 1786-1797. (2007)

3. Hajimohammadi, M., Safari, N., Mofakham, H., Shaabani, A.: A new and efficient aerobic oxidation of aldehydes to carboxylic acids with singlet oxygen in the presence of porphyrin sensitizers and visible light. Tetrahedron Letters. 51 4061-4065. (2010)

4. Bennett, L.E., Ghiggino, K.P., Henderson, R.W.: Singlet oxygen formation in monomeric and aggregated porphyrin. Journal of Photochemistry and Photobiology B: Biology. 3 81-89. (1989)

5. Christoforidis, K., Louloudi, M, Milaeva, E., Deligiannakis, Y.: Mechanism of catalytic decomposition of pentachlorophenol by a highly recyclable heterogeneous $\mathrm{SiO}_{2}-[$ Fe-porphyrin] catalyst. Journal of Catalysis. 270 153-162. (2010)

6. Cai, J.H., Huang ,J.W, Yu, H.C, Ji, L.N.: Fabrication and characterizations of silica composite microspheres immobilized with porphyrins and their photocatalytic properties. Journal of the Taiwan Institute of Chemical Engineers. 43 958-964. (2012)

7. García-Sánchez, M.A., de la Luz, V., Coahuila-Hernández, M.I., Rojas-González, F., Tello-Solís, S.R., Campero, A.: Effects of the structure of entrapped substituted porphyrins on the textural characteristics of silica networks. Journal of Photochemistry and Photobiology A: Chemistry. 223 172-181. (2011)

8. Li, X., Zhang, L., Mu, J.: Synthesis and characterization of silica microspheres functionalized with porphyrin monolayer. Applied Surface Science. 253 525-529. (2006)

9. Papacídero, A., Rocha, L., Caetano, B., Molina, E., Sacco, H., Nassar, E., Martinelli, Y., Mello, C., Nakagaki, S., Ciuffi, K.: Preparation and characterization of 
spherical silica-porphyrin catalysts obtained by the sol-gel methodology. Colloids and Surfaces A: Physicochemical and Engineering Aspects. 275 27-35. (2006)

10. Rollman, L.D.: Porous, polymer-bonded metalloporphyrins. J. Am. Chem. Soc. 97 2132-2136. (1975)

11. Cooke, P.R., Linsay, J.R.: Alkene epoxidation catalysed by ligand-bound supported metalloporphyrins. Tetrahedron Lett. 33 2737-2740. (1992)

12. Brulé, E., de Miguel, Y.R.: Supported metalloporhyrin catalysts for alkene epoxidation. Org Biomol Chem. 4 599-609. (2006)

13. Battioni, P., Lallier, J.P., Barloy, L., Mansuy, D.: Mono-oxygenase-like oxidation of hydrocarbons using supported manganese-porpnyrin catalysts: beneficial effects of a silica support for alkane hydroxylation. J. Chem. Commun. 109 1149-1151. (1989)

14. Nakamura, M., Tatsumi, T., Tominaga, H.: Synthesis and Catalytic Capability of zeolite-Encapsulated Iron and Manganese tetramethylporphine complexes. Bull. Chem. Soc. Jpn. 63 3334-3336. (1990)

15. Papacídero, A., Rocha, L., Caetano, B., Molina, E., Sacco, H., Nassar, E., Martinelli, Y., Mello, C., Nakagaki S., Ciuffi, K.: Preparation and characterization of spherical silica-porphyrin catalysts obtained by the sol-gel methodology. Colloids and Surfaces A: Physicochem. Eng. Aspects. 275 27-35. (2006)

16. Monsalves, M., P. Martins, Curi, R., Nascimento, O., Iamamoto, Y.: Iron porphyrins immobilised on silica surface and encapsulated in silica matrix: a comparison of their catalytic activity in hydrocarbon oxidation. Journal of Molecular Catalysis A: Chemical. 233 73-81. (2005)

17. García-Sánchez. M.A, de la Luz V., Coahuila-Hernández. M.I., Rojas-González. F., Tello-Solís S.R., Campero A.: Effects of the structure of entrapped substituted porphyrins on the textural characteristics of silica networks. Journal of Photochemistry and Photobiology A: Chemistry. 223 172-181. (2011)

18. García-Sánchez, M.A, Campero, A.: Cobalt ortho- and para-substituted tetraphenylporphyrins inserted in $\mathrm{SiO}_{2}$ gels. Journal of Non-Crystalline Solids. 333 226-230. (2004)

19. Adler, A.D., Longo, F.R., Kampas, F., Kim, J.: On the preparation of metalloporphyrins. J. Inorg. Nucl. Chem. 32 2443-2445. (1970)

20. López, T., López-Gaona, A., Gómez, R.: Synthesis, characterization and activity of $\mathrm{Ru} / \mathrm{SiO}_{2}$ catalysts prepared by the sol-gel method. J. Non-Cryst. Solids. 110 170-174. (1989)

21. Gouterman, M., Wagniere, G.H., Snyder, L.C.: The porphyrins. J. Mol. Spectrosc. 11, 108. (1963)

22. Zheng, W., Shan, N., Yu, L., Wang, X.: UV-visible, fluorescence and EPR properties of porphyrins and metalloporphyrins. Dyes and Pigments. 77 153-157. (2008)

23. Takagi, S., Shimada, T., Yui, T., Inoue, H.: High Density Adsorption of Porphyrins onto Clay Layer without Aggregation: Characterization of Smectite-Cationic Porphyrin Complex. Chem.Lett. 30 128-129. (2001)

24. Cady, S.S., Pinnavaia, T.J.: Porphyrin intercalation in mica-type silicates. Inorg. Chem. 17 1501-1507. (1978) 